\title{
Serum bilirubin concentration as a marker of severity of acute appendicitis
}

\author{
Maciej Jastrzębski ${ }^{1}$, Maciej Krasnodębski ${ }^{1}$, Michalina Szczęśniak ${ }^{1}$, Michał Wierzchowski ${ }^{1}$, Julia Pikul ${ }^{1}$, \\ Dariusz Jabłoński ${ }^{2}$, Michał Grąt ${ }^{1}$, Tadeusz Wróblewski ${ }^{1}$, Krzysztof Zieniewicz ${ }^{1}$ \\ ${ }^{1}$ Department of General, Transplant and Liver Surgery, Medical University of Warsaw, Warsaw, Poland \\ ${ }^{2}$ Public Central Teaching Hospital, Warsaw, Poland
}

Videosurgery Miniinv 2020; 15 (1): 117-122

DOI: https://doi.org/10.5114/wiitm.2019.87140

\begin{abstract}
Introduction: Acute appendicitis $(A A)$ is one of the most common causes of urgent admission to the hospital. Clinically applicable classification distinguishes simple and complex inflammation. Among commonly used inflammation markers of $A A$, bilirubin concentration is not well studied and thus is rarely applied.

Aim: To examine the association between increased serum total bilirubin concentration and the severity of $A A$.

Material and methods: This retrospective study included 169 patients with a presumptive diagnosis of $A A$ who were operated upon between 2015 and 2017. The determined study endpoints were simple complex inflammation and a different diagnosis after surgery. The Mann-Whitney U, Kruskal-Wallis, Fisher's exact, Spearman correlation coefficient and logistic regression tests and receiver-operating characteristics (ROC) were used in analyses. The area under the curve (AUC) was presented with 95\% confidence intervals (95\% Cls). Statistical significance was set at 0.05.

Results: In total, 84 (49.7\%) patients underwent laparotomy and 85 (50.3\%) laparoscopy. After surgery, 45 (26.6\%) patients had a diagnosis other than AA. Furthermore, 83 (49.1\%) and 41 (24.3\%) patients had simple and complex $A A$, respectively. The median bilirubin concentration was $0.56,0.69$, and $1.08 \mathrm{mg} / \mathrm{dl}$ in patients without $A A$, with simple, and complex $A A$, respectively $(p<0.01)$. The optimal cut-off for serum bilirubin concentration to predict $A A$ severity was $\geq 0.94 \mathrm{mg} / \mathrm{dl}$ ( $A \cup C=0.652 ; 95 \% \mathrm{Cl}: 0.543-0.761)$ with a $44.9 \%$ positive and $83.9 \%$ negative predictive value $(p=0.006)$

Conclusions: The serum bilirubin concentration should be considered as one of the possible markers of AA. Moreover, it can be used to predict the severity of $A A$.
\end{abstract}

Key words: laparoscopic appendectomy, acute appendicitis, serum bilirubin concentration, complex appendicitis.

\section{Introduction}

Acute appendicitis (AA) is one of the most common emergencies [1-3] and is the reason for 10$20 \%$ of all acute surgical admissions for abdominal pain [4]. Likewise, appendectomies remain among the most frequently performed emergency surgical procedures $[1,5]$. Its lifetime risk and incidence are estimated to be $7-8 \%[1,6]$ and 88/100 000 per year, respectively [2]. Despite the high prevalence, this condition is commonly misdiagnosed [7], with a negative appendectomy rate as high as $10-40 \%$ according to previous studies [8, 9].

The lack of reliable and unambiguous markers for $A A$ is cited as the main reason for the false-positive diagnoses $[1,5]$. Therefore, the diagnostic approach is still based on the clinical presentation and laboratory tests (white blood cells, C-reactive protein), which

\section{Address for correspondence}

Maciej Krasnodebski MD, PhD, Department of General, Transplant and Liver Surgery, Medical University of Warsaw, 1 A Banacha St,

02-097 Warsaw, Poland, phone: +48 2259925 45, fax: +48 22599 15 45, e-mail: mwkrasn@gmail.com 
have limited accuracy even if combined with scoring systems (such as the Alvarado score) [1, 5, 10-12]. Lately, the sensitivity improved due to the introduction of imaging studies, such as ultrasound and computed tomography (CT), into the diagnostic pathway, although these also have their limitations. A correct, detailed interpretation of the ultrasound scan results requires an experienced diagnostician, whereas the prevalent use of CT would increase the cost and contribute to abundant radiation exposure $[1,11]$.

To find a specific marker for AA, recent studies considered an association between an increase in serum total bilirubin concentration (TBil) and the development of complex (gangrenous or perforated) AA $[7,10,13,14]$. Other studies have attempted to evaluate the usefulness of hyperbilirubinemia in distinguishing AA from other conditions presenting with similar clinical symptoms, and the outcomes confirmed the hypothesis $[2,15]$. Moreover, in the face of growing evidence for non-surgical treatment of uncomplicated AA the search for a clinically useful marker to establish the severity of AA seems obligatory [16].

\section{Aim}

The aim of the present study was to examine the association between increased serum total bilirubin concentration and the development of complex AA (concurrent with perforation and/or necrosis) [13].

\section{Material and methods}

This retrospective study involved all patients admitted to the Surgical Department between January 2015 and December 2017 with clinical presentations of AA. Collected data covered patients' laboratory values, results of imaging studies, body mass index (BMI; height and weight), and reports regarding complications and treatment during the hospital stay. A total of 169 patients with a presumptive diagnosis of AA who underwent an operation were included in this study. Patients were scheduled to undergo emergency laparotomy/laparoscopy either by elevated inflammatory markers and/or imaging studies with suspicion of acute appendicitis or by peritonitis signs during physical examination. Prior to admission, female patients were consulted by a gynecologist and those in reproductive age also had blood tests for human chorionic gonadotropin level.
The patients were then classified into 3 consecutive groups according to appendix histopathology and operative reports. The first group comprised patients with simple inflammation understood as simple and suppurative AA. The second group included patients with complex and thus gangrenous or perforated AA. Patients with a final diagnosis other than AA constituted the third group.

\section{Statistical analysis}

The Kruskal-Wallis and Mann-Whitney $U$ tests, Fisher's exact test, Spearman correlation coefficient and logistic regression were used. A $p$-value $<0.05$ was considered to be statistically significant. Confidence intervals (Cls) were set at $95 \%$. A receiver operating characteristics (ROC) curve was plotted for perforated and simple appendicitis in terms of serum bilirubin concentration. The laboratory norm for total bilirubin concentration in our hospital is set at $<1.2 \mathrm{mg} / \mathrm{dl}$. Analyses were computed using Statistica version 13.1 software (Dell Inc., Tulsa, USA). The study protocol was approved by the local ethics committee and has therefore been performed in accordance with the Helsinki Declaration of 1975.

\section{Results}

Patients' characteristics are shown in Table I. Appendicitis was confirmed histologically in 124 of 169 patients (73.4\%). Eighty-three patients were diagnosed with simple AA ( $49.1 \%$ of all), and the remaining 41 (24.3\%) patients developed complex inflammation. The median serum total bilirubin concentration for all 3 groups was $0.56,0.69$, and 1.08 $\mathrm{mg} / \mathrm{dl}$ for non-appendicitis, simple AA, and complex $A A$, respectively $(p=0.020)$ (Figure 1$)$. The $C$-reactive protein (CRP) concentration was positively correlated with TBil $(R=0.271, p<0.001)$ in all patients. The optimal cut-off point for TBil for prediction of complex appendicitis was $0.94 \mathrm{mg} / \mathrm{dl}$ (Figure 2) with a sensitivity of $59.5 \%$, specificity of $74.3 \%$, positive predictive value (PPV) of $44.9 \%$, and negative predictive value (NPV) of $83.9 \%$. The area under the curve (AUC) was 0.652 (95\% Cl: 0.543-0.761), $p=$ 0.006 . The ROC curve analysis for estimation of simple AA was not statistically significant $(p=0.067)$. Analyses of the percentage of patients with complex appendicitis with hyperbilirubinemia compared to the rest of the patients with increased TBil (with simple appendicitis or without it) were statistically 
Table I. Baseline characteristics of the study group

\begin{tabular}{|c|c|}
\hline Parameter & $\begin{array}{l}\text { Median (interquartile } \\
\text { range) or quantity } \\
\text { (percent) }\end{array}$ \\
\hline Male & $84(49.7 \%)$ \\
\hline Laparoscopic appendectomy & $85(50.3 \%)$ \\
\hline Age [years] & $33.0(27.0-49.0)$ \\
\hline $\mathrm{BMI}\left[\mathrm{kg} / \mathrm{m}^{2}\right]$ & $23.9(20.7-27.2)$ \\
\hline WBC $\left[10^{3} / \mu l\right]$ & $12.6(9.9-15.8)$ \\
\hline $\mathrm{NEU}\left[10^{3} / \mu \mathrm{l}\right]$ & $9.7(6.9-13.1)$ \\
\hline $\mathrm{HGB}[\mathrm{g} / \mathrm{dl}]$ & $13.9(13.0-15.2)$ \\
\hline TBil [mg/dl] & $0.7(0.5-1.2)$ \\
\hline CREA $[\mathrm{mg} / \mathrm{dl}]$ & $0.9(0.7-1.0)$ \\
\hline $\operatorname{CRP}[\mathrm{mg} / \mathrm{l}]$ & $23.9(5.0-79.4)$ \\
\hline AST $[U / I]$ & $20.0(17.0-28.0)$ \\
\hline ALT $[U / I]$ & $24.0(20.0-33.0)$ \\
\hline Amylase [U/l] & $45.0(32.0-56.0)$ \\
\hline Fibrinogen [mg/dl] & $375.0(306.0-466.0)$ \\
\hline INR & $1.06(1.00-1.15)$ \\
\hline
\end{tabular}

$B M I$ - body mass index, WBC - white blood cells, NEU - neutrophils, HGB hemoglobin, TBil - total bilirubin, CREA - creatine, CRP - C-reactive protein, $A S T$ - aspartate transferase, ALT - alanine transferase, INR - international normalized ratio of the prothrombin time.

significant $(p=0.003)$ with sensitivity of $43.2 \%$ and specificity of $82.9 \%$. Analyses of the percentage of patients with hyperbilirubinemia with or without appendicitis (either form) were not statistically significant $(p=0.830)$. The increase in the patients' TBil, white blood cells (WBC), and CRP concentrations is displayed in Table II. Multivariable analysis revealed

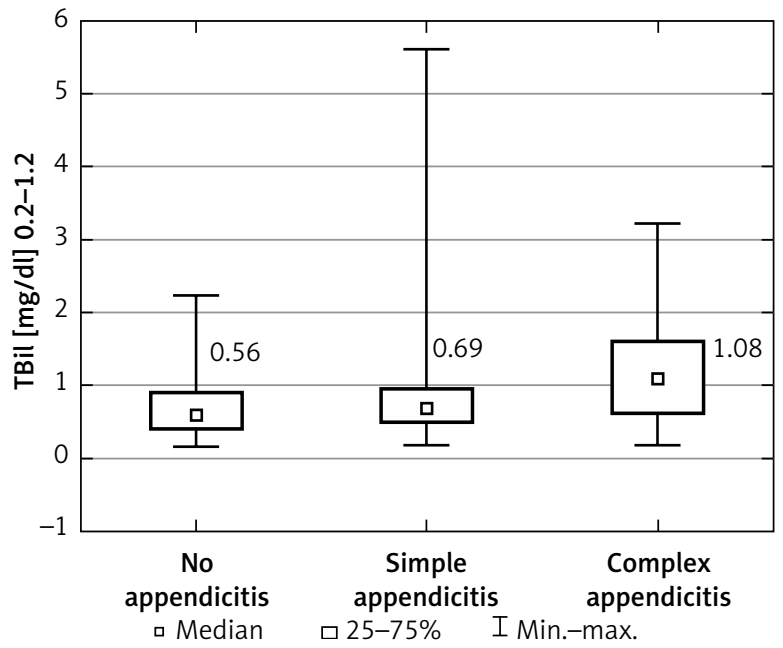

Figure 1. Box plot showing the median serum bilirubin concentration in regard to the groups

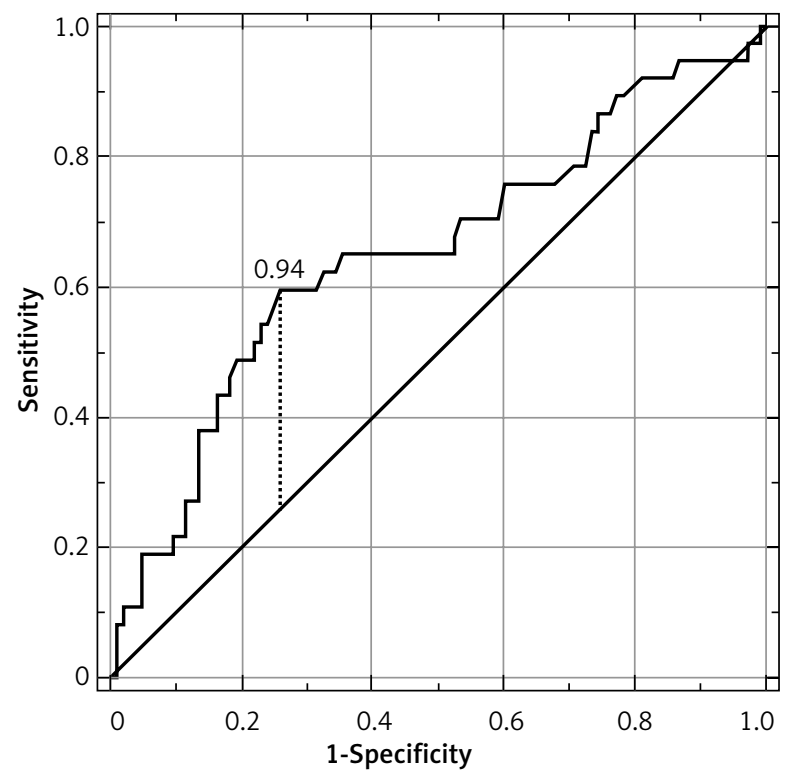

Figure 2. ROC curve with optimal cut-off point for serum bilirubin concentration

Table II. Quantity of patients with increased biochemical markers and patients with increased bilirubin above optimal cut-off point established by ROC analysis

\begin{tabular}{|lccc|}
\hline Parameter & No appendicitis & Simple appendicitis & Complex appendicitis \\
\hline $\begin{array}{l}\text { TBil }(n=142) \\
\geq 0.94[\mathrm{mg} / \mathrm{dll}]\end{array}$ & 10 of $41(24.4 \%)$ & 17 of $64(26.6 \%)$ & 22 of $37(59.5 \%)$ \\
\hline $\begin{array}{l}\text { TBil }(n=142) \\
>1.2[\mathrm{mg} / \mathrm{dl}]\end{array}$ & 9 of $41(26.5 \%)$ & 9 of $64(14.06 \%)$ & 16 of $37(43.2 \%)$ \\
\hline $\begin{array}{l}\text { WBC }(n=169) \\
>11\left[10^{3} / \mathrm{ml}\right]\end{array}$ & 25 of $45(55.6 \%)$ & 54 of $83(65.1 \%)$ & 33 of $41(80.5 \%)$ \\
\hline $\begin{array}{l}\text { CRP }(n=169) \\
>10[\mathrm{mg} / \mathrm{l}]\end{array}$ & 25 of $45(55.6 \%)$ & 48 of $83(57.8 \%)$ & 35 of $41(85.4 \%)$ \\
\hline
\end{tabular}

TBil - total bilirubin, WBC - white blood cells, CRP - C-reactive protein. 
Table III. Multivariable analysis of factors independently associated with complex AA

\begin{tabular}{|lcc|}
\hline \multirow{2}{*}{ Parameter } & \multicolumn{2}{c|}{ Multivariable } \\
\cline { 2 - 3 } & OR $(95 \% \mathrm{Cl})$ & $P$-value \\
\hline $\mathrm{CRP}^{*}[\mathrm{mg} / \mathrm{l}]$ & $1.10(1.03-1.17)$ & 0.001 \\
\hline TBil $\geq 0.94 \mathrm{mg} / \mathrm{dl}$ & $1.67(1.01-2.59)$ & 0.020 \\
\hline WBC $\left[10^{3} / \mu \mathrm{l}\right]$ & $1.11(1.01-1.23)$ & 0.040 \\
\hline
\end{tabular}

Odds ratios for quantitative variables are given per units specified in brackets. CRP - C-reactive protein, TBil - total bilirubin concentration, WBC white blood cells. *For every 10 units.

Table IV. Conditions other than AA of gynecological origin

\begin{tabular}{|ll|}
\hline Adnexitis & 6 \\
\hline Ovarian cyst rupture & 3 \\
\hline Tumor of the right ovary & 1 \\
\hline Suspicion of endometriosis & 1 \\
\hline
\end{tabular}

that TBil above $0.94 \mathrm{mg} / \mathrm{dl}$ was an independent predictor for complex AA among WBC and CRP (Table III). The overall postoperative complication rate for all patients was $13.6 \%$ (9.3\% in simple AA, $29.3 \%$ in complex and $6.7 \%$ in non-AA group). Postoperative complications were not significantly correlated with bilirubin concentration ( $p=0.525$ ).

Other conditions that affected patients primarily diagnosed with AA were gynecological conditions ( $n=11$; displayed in Table IV), lymphadenitis ( $n=6$ of which 4 were mesenteric lymphadenitis), inflammation of the surrounding soft tissues $(n=6)$, mucinous cystadenoma of the appendix $(n=3)$, inflammation associated with a G1 NET tumor $(n=2)$, and chronic inflammation of the appendix $(n=2)$. Apart from that a single case of cholecystitis, infiltrating cecal tumor, Meckel's diverticulitis, stomach perforation, colon diverticulitis infiltrating the appendix, adhesive bowel disease, appendiceal cyst, infiltration of the lymphoma, and abdominal pain 10 months after caesarean section were noted. The cause of symptoms was not found in 6 patients. Seventy-one patients developed periappendicitis.

\section{Discussion}

Impairment of hepatic function as a result of septic conditions has been well described [2]. Jaundice was observed in patients with AA over 60 years ago [5].
The putative mechanism of hyperbilirubinemia in AA is associated with bacteria and bacterial toxins carried by the portal vein to the liver. Both the cytotoxicity of the bacterial product and the inflammatory response lead to dysfunction of the hepatocytes, which affects bilirubin secretion [2, 15, 17]. Other studies [7] have indicated that hemolysis, caused by a septic condition, is an important mechanism causing hyperbilirubinemia. This statement explains the lack of increase in liver transaminases in $95-98 \%$ of the patients $[2,18]$.

Recently, an increasing amount of studies have examined the association between hyperbilirubinemia and inflammation of the vermiform appendix, some of which found bilirubin to be a specific marker for appendiceal perforation. According to D'Souza et al. [2], Muller et al. [7], Giordano et al. [10] and Silva et al. [19] the specificity, sensitivity, PPV, and NPV of hyperbilirubinemia compared for simple versus perforated appendicitis were $70-82 \%[2,7,10,19]$, $48-70 \%[2,7,10,19], 18-47 \%[2,7]$, and $92-93 \%$ $[2,7]$, respectively. For comparison, results pooled in this research were $74.3 \%, 59.5 \%, 44.9 \%$, and $83.9 \%$, respectively. Whereas the specificity, sensitivity and PPV nearly matched those found in the literature [2, 7, 10, 19] the NPV was slightly lower.

With regards to final alternative diagnoses, the most common were neoplasms and gynecological conditions as well as other inflammatory aliments such as adnexitis or lymphadenitis. A common co-existing morbidity with AA pathology was periappendicitis, defined as inflammation of the surrounding soft tissue (neutrophilic infiltration). A probable explanation for the suspicion of appendicitis is the misleading signs of peritonitis caused mostly by free blood in the abdomen. Even a small amount of blood can cause irritation of the peritoneum. Second, easy access and abuse of antibiotics may alleviate the symptoms of appendicitis and lower inflammation markers prior to hospital admission in an emergency. In such cases, the distinction of acute appendicitis by surgeons becomes more and more difficult in our time.

Evidence suggests that laparoscopic appendectomy appears to be a method of choice in most patients presenting with the symptoms of AA [6, 16]. However, recent studies such as the APPAC randomized clinical trial published by Salminen et al. [20] and a meta-analysis by Podda et al. [16], which included more than 3500 patients with suspicion of $A A$, have shown that antibiotic therapy for image-proven uncomplicated appendicitis is a viable 
treatment option compared to surgery. Podda et al. [16] reported antibiotic treatment to have a failure rate of $27.7 \%$ at 1 -year follow-up along with lower complication-free treatment success (no postoperative complications, adverse events, or treatment failure occurring $-67.2 \%$ for conservative vs. $82.3 \%$ for surgical management). Additionally, a decreased rate of postintervention complications (7.1\% vs. $14.5 \%$ ) and reduced cost were noted. They also found that the differences between patients with uncomplicated AA treated primarily surgically and conservatively, who required a second hospitalization due to recurrent appendicitis, were not statistically significant, as far as increased perforation rate and the higher risk of postoperative complications were concerned. Currently the only appreciable examination to differentiate simple and complex AA is CT $[20,21]$, but its use in every patient cannot be justified as it requires exposure to a considerable dose of radiation along with being high in cost for the hospital [3].

The study has several limitations. Firstly, it is a retrospective study with a moderate number of patients. Secondly, due to the retrospective nature of the study, the total bilirubin concentration could not be examined for the division of its components (unconjugated and conjugated). Lastly, there is also a concern regarding potential inclusion of patients with Gilbert's syndrome in the study group. On the one hand, it could influence outcomes. On the other hand, inclusion of those patients corresponds to a more natural situation. Not all patients are aware of having Gilbert's syndrome and there are no methods to confirm this on the day of the emergency admittance to the hospital.

\section{Conclusions}

As far as the utility for serum bilirubin concentration in the diagnoses of complex AA is concerned, the results were equivocal. On the one hand, the poor sensitivity and PPV indicated that as a standalone marker, serum bilirubin concentration is not enough to predict a perforation; on the other hand, the obtained high negative predictive value can help in the selection of patients who do not require immediate surgery. Some of them can be treated conservatively and/or subjected to further observation. Owing to a satisfactory specificity, a presumptive diagnosis of complex AA can be confirmed. Undoubtedly, an increase in TBil is not an anomalous biochemical test outcome in AA and should be considered by physicians as one of the markers in a more severe condition. Bilirubin can be used as an additional indicator enabling the selection of a group of patients who will not require urgent surgery. This marker is relatively cheap and available in every emergency department, and its value turns out to be clinically useful.

\section{Conflict of interest}

The authors declare no conflict of interest.

\section{References}

1. Bhangu A, Srrreide K, Di Saverio S, et al. Acute appendicitis: modern understanding of pathogenesis, diagnosis, and management. Lancet 2015; 386: 1278-87.

2. D'Souza N, Karim D, Sunthareswaran R. Bilirubin: a diagnostic marker for appendicitis. Int J Surg 2013; 11: 1114-7.

3. Acharya A, Markar SR, Ni M, et al. Biomarkers of acute appendicitis: systematic review and cost-benefit trade-off analysis. Surg Endosc 2017; 31: 1022-31.

4. Fagerström A, Paajanen P, Saarelainen H, et al. Non-specific abdominal pain remains as the most common reason for acute abdomen: 26-year retrospective audit in one emergency unit. Scand J Gastroenterol 2017; 52: 1072-7.

5. Al-Abed YA, Alobaid N, Myint F. Diagnostic markers in acute appendicitis. Am J Surg 2015; 209: 1043-7.

6. Lasek A, Pędziwiatr M, Wysocki M, et al. Risk factors for intraabdominal abscess formation after laparoscopic appendectomy - results from the Pol-LA (Polish Laparoscopic Appendectomy) multicenter large cohort study. Videosurgery Miniinv 2019; 14: 70-8.

7. Muller S, Falch C, Axt S, et al. Diagnostic accuracy of hyperbilirubinaemia in anticipating appendicitis and its severity. Emerg Med J 2014; 0: 1-5.

8. Sadot E, Wasserberg N, Shapiro R, et al. acute appendicitis in the twenty-first century: should we modify the management protocol? J Gastrointest Surg 2013; 17: 1462-70.

9. Xingye W, Yuqiang L, Rong W, Hongyu Z. Evaluation of diagnostic scores for acute appendicitis. J Coll Physicians Surg Pak 2018; 28: 110-4.

10. Giordano S, Pääkkönen M, Salminen P, et al. Elevated serum bilirubin in assessing the likelihood of perforation in acute appendicitis: a diagnostic meta-analysis. Int J Surg 2013; 11: 795-800.

11. Ozkan S, Duman A, Durukan P, et al. The accuracy rate of Alvarado score, ultrasonography, and computerized tomography scan in the diagnosis of acute appendicitis in our center. Niger J Clin Prac 2014; 17: 413-8.

12. Erdem H, Çetinkünar S, Daş K, et al. Alvarado, Eskelinen, Ohhmann and Raja Isteri Pengiran Anak Saleha Appendicitis scores for diagnosis of acute appendicitis. World J Gastroenterol 2013; 19: 9057-62.

13. Chaudhary P, Kumar A, Saxena N, et al. Hyperbilirubinemia as a predictor of gangrenous/perforated appendicitis: a prospective study. Ann Gastroenterol 2013; 26: 325-31. 
14. Jamaluddin M, Abbas Hussain SM, Ahmad H. Hyperbilirubinaemia a predictive factor for complicated acute appendicitis: a study in a tertiary care hospital. J Pakistan Med Assoc 2013; 63: 1374.

15. Sandstrom A, Grieve DA. Hyperbilirubinaemia: its utility in non-perforated appendicitis. ANZ J Surg 2015; 87: 587-90.

16. Podda M, Gerardi C, Cillara N, et al. Antibiotic treatment and appendectomy for uncomplicated acute appendicitis in adults and children: a systematic review and meta-analysis. Ann Surg 2019 doi: 10.1097/SLA.0000000000003225.

17. Woźnica EA, Inglot M, Woźnica RK, et al. Liver dysfunction in sepsis. Adv Clin Exp Med 2018; 27: 547-51.

18. Estrada JJ, Petrosyan M, Barnhart J, et al. Hyperbilirubinemia in appendicitis: a new predictor of perforation. J Gastrointest Surg 2007; 11: 714-8.

19. Silva FR, da Rosa MI, Silva BR, et al. Hyperbilirubinaemia alone cannot distinguish a perforation in acute appendicitis. ANZ J Surg 2015; 86: 255-9.

20. Salminen P, Paajanen H, Rautio T, et al. Antibiotic therapy vs appendectomy for treatment of uncomplicated acute appendicitis. The APPAC Randomized Clinical Trial. JAMA 2015; 313 2340-8.

21. Varadhan KK, Neal KR, Lobo DN. Safety and efficacy of antibiotics compared with appendicectomy for treatment of uncomplicated acute appendicitis: meta-analysis of randomised controlled trials. BMJ 2012; 344: e2156.

Received: 14.06.2019, accepted: 6.07.2019. 